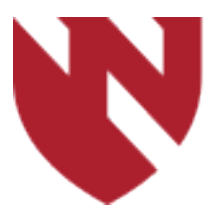

September 2020

\title{
The Clinical Relevance of Cement Volume in Percutaneous Vertebral Augmentation
}

\author{
Michael Warden \\ University of Nebraska Medical Center \\ Ravi G. Mirpuri \\ University of Nebraska Medical Center \\ Virginia Hardie \\ University of Nebraska Medical Center \\ Madhuri Are \\ University of Nebraska Medical Center
}

Tell us how you used this information in this short survey.

Follow this and additional works at: https://digitalcommons.unmc.edu/gmerj

Part of the Higher Education Commons, and the Medicine and Health Sciences Commons

\section{Recommended Citation}

Warden, M., Mirpuri, R. G., Hardie, V., , Are, M. The Clinical Relevance of Cement Volume in Percutaneous Vertebral Augmentation. Graduate Medical Education Research Journal. 2020 Sep 29; 2(1). https://digitalcommons.unmc.edu/gmerj/vol2/iss1/29

This Conference Proceeding is brought to you for free and open access by DigitalCommons@UNMC. It has been accepted for inclusion in Graduate Medical Education Research Journal by an authorized editor of DigitalCommons@UNMC.For more information, please contact digitalcommons@unmc.edu. 


\section{The Clinical Relevance of Cement Volume in Percutaneous Vertebral Augmentation}

\section{Creative Commons License}

\section{(c) (1) $\Theta(9$}

This work is licensed under a Creative Commons Attribution-Noncommercial-No Derivative Works 4.0 License. 


\section{Sleep Improvement Program and Academic Performance}

Matthew Tingle ${ }^{1}$, Liz Wellsandt ${ }^{2}$, Kari Nelson ${ }^{3}$, RJ Barber ${ }^{1}$, Beau Konigsberg ${ }^{1}$, Maxwell Lydiatte ${ }^{4}$, Cale Ewald ${ }^{4}$, Matthew McManigal ${ }^{2}$, Matthew Tao ${ }^{1}$

1 University of Nebraska Medical Center, Department of Orthopedic Surgery

${ }^{2}$ University of Nebraska Medical Center, College of Allied Health Professions, Physical Therapy

${ }^{3}$ University of Nebraska Medical Center, Office of Graduate Medical Education

${ }^{4}$ University of Nebraska Medical Center, College of Medicine

\section{Mentor: Matthew Tao}

Program: Orthopedic Surgery

Type: Original Research

Background: The aim of this study is to investigate the impact of improved sleep quality and extension can have on academic performance in medical students. Additional outcome measures include evaluating mood (Profile of Mood States) and sleepiness (Epworth Sleepiness Scale). Our hypothesis is that improved sleep quality and extension will significantly improve test scores, mood and daytime sleepiness.

Methods: This is a pilot study involving 20 first-year medical students. They will be randomized into either control or intervention groups. Both groups will be monitored for baseline sleep characteristics. The intervention group will then undergo a training program aimed to improve sleep quality and extension. Subjects will be monitored via actigraphy devices (Actigraph wGT3x-BT) on the dominant hand at all times as well as daily, subjective sleep logs. The primary outcome will be raw test scores at the typical testing intervals based on the curriculum. ESS questionnaire will be administered at the beginning and end of the study, and the POMS will be administered weekly.

Results and Conclusion: Pending https://doi.org/10.32873/unmc.dc.gmerj.2.1.027

\section{The Clinical Relevance of Cement Volume in Percutaneous Vertebral Augmentation} Ravi G. Mirpuri', Michael J. Warden'1, Virginia Hardie', Madhuri Are ${ }^{1}$

${ }^{1}$ University of Nebraska Medical Center, Department of Anesthesiology

\section{Mentor: Madhuri Are}

Program: Anesthesiology

Type: Original Research

Background: Vertebroplasty and Kyphoplasty are two forms of percutaneous vertebral augmentation (PVA), in which polymethylmethacrylate cement is used to stabilize vertebral compression fractures (VCF). This study sought to evaluate the relationship between cement volume and clinical outcomes, including pain reduction, opioid use, and complication rate.

Methods: Retrospective chart review produced 88 patients who received PVA at a tertiary care outpatient pain clinic. Cement volume, type of PVA, gender, level (thoracic vs lumbar) were collected, as well as clinical outcomes of numeric pain score (NPS) reduction, opioid percent change (OPC), and complications. Both pre-procedure and postprocedure (between 2-4 weeks) data were collected.

Results: Sixty-four patients (72.7\%) had statistically significant NPS reduction of $\geq 50 \%$ pain $(\mathrm{p}<.001)$. The mean NPS reduction was $4.45(\mathrm{p}<.0001)$. Also sixtythree out of seventy-two patients who previously used opioids demonstrated some form of opioid reduction which was significant $(\mathrm{p}<.0001)$. The mean opioid reduction measured across all baseline opioid users was $48 \%$. No statistically significant differences in NPS or OPC were seen across gender or level type. Linear regression using pre NPS as a covariate showed cement volume did not have a significant effect on NPS change or opioid reduction $(\mathrm{p}=0.173$ and $\mathrm{p}=.299$ respectively). Cement leakage occurred as a complication in $10.2 \%$ of the patients, although only one complication was symptomatic.

Conclusion: Cement volume does not correlate with clinical outcomes of NPS reduction or OPC. Furthermore, our study reinforced PVA as a beneficial treatment for reducing pain and opioid consumption in patients with painful VCF.

https://doi.org/10.32873/unmc.dc.gmerj.2.1.028

\section{Diagnostic Quality and Radiation Dose for Pulmonary Embolism Protocol Computed Tomography: ASIR-V vs. Standard Filtered Back Projection \\ Lei Yu ${ }^{1 *}$, Antonia Gurney ${ }^{1 *}$, Kelly Dea ${ }^{1 *}$, Neil Hansen ${ }^{1}$, Valerie Shostrom², Chandrakanth Are ${ }^{3}$, Anna Zajicek $^{1}$ \\ ${ }^{1}$ University of Nebraska Medical Center, Department of Radiology \\ ${ }^{2}$ University of Nebraska Medical Center, College of Public Health, Department of Biostatistics \\ ${ }^{3}$ University of Nebraska Medical Center, Office of Graduate Medical Education \\ *authors contributed equally to the work}

Mentor: Anna Zajicek

Program: Radiology

Type: Review/Meta-analysis

Background: Pulmonary embolism (PE) is the third leading cause of acute cardiovascular disease, contributes to 250,000 American hospitalizations yearly. CT pulmonary angiography (CTPA) is the first-line imaging choice for suspected PE, however radiation exposure remains a concern. We compared the Dose-Length Product (DLP) in CTPA performed using adaptive statistical iterative reconstruction - V (ASIR-V) versus older filtered back projection (FBP) technique, while also evaluating the ability of ASIR-V to maintain diagnostic accuracy.

Methods: A retrospective review was performed of all CTPA scans completed for suspected PE during a one-year period at our institution. A total of 1095 studies met criteria for inclusion. Five-hundred and thirty-three $(48.7 \%)$ of these were performed on a CT scanner utilizing ASIR-V (GE Healthcare), and $562(51.3 \%)$ were performed on an older CT scanner utilizing standard FBP (Lightspeed VCT, GE Healthcare). Doselength product (DLP) and demographic data were recorded. Original radiology reports from board-certified radiologists for each exam were reviewed. Diagnostic quality was determined by usage of specified 